ketamine in group PK was $1.49(0.61) \mathrm{mg} / \mathrm{hg} / \mathrm{h}$. There were no significant differences in patient tolerance, discomfort during insertion, patient and endoscopist satisfaction, haemodynamic responses, procedural pain, recovery time and recovery score. Overall, cardiovascular and respiratory adverse events were not significantly different between the two groups. These adverse events were transient and easily treated with no sequelae.

Conclusion IVS in both regimens provided effective and safe for colonoscopy. Adverse events were relatively high in both groups. However, these adverse events were mild and transient. No serious adverse events were observed.

Competing interests None declared.

\section{PWE-106 IS IT NECESSARY FOR ALL COLORECTAL CANCER PATIENTS WITH LIVER METASTASIS TO BE DISCUSSED AT A HEPATOBILIARY MDT?}

doi:10.1136/gutjnl-2012-302514d.106

${ }^{1} \mathrm{~S}$ Vasudevan, ${ }^{*}{ }^{1} \mathrm{~J}$ Wright, ${ }^{2} \mathrm{M}$ Rees, ${ }^{2} \mathrm{~B}$ Creswell, ${ }^{1} \mathrm{D}$ Stiff, ${ }^{1} \mathrm{~A}$ Wordley, ${ }^{1} \mathrm{R}$ Motson. ${ }^{1}$ Colorectal Surgery, Colchester University Hospital, Colchester, UK; ${ }^{2}$ Hepatobiliary Surgery, Pelican Cancer Foundation, Basingstoke, UK

Introduction Current guidelines suggest an opinion from a hepatobiliary MDT should be sought for all patients with colorectal cancer liver metastasis. This places a significant burden of work on the hepatobiliary MDT who are often the referral centre for many hospitals (Approximately 20 cases per month at our referral centre). This study was performed to see if our local colorectal MDT was able to make a correct decision regarding referral for consideration of liver resection by comparing its decision with the decision from two hepatobiliary surgeons from our referral network.

Methods CT scans from 38 patients found to have liver metastasis from colorectal cancer were anonymised and sent to two hepatobiliary surgeons in our cancer network (Pelican Centre, Basingstoke). They classified them into three categories; U-Unresectable, CChemo to downstage then consider resection and $\mathrm{R}-$ Resectable. The results were then compared with the opinion of our local colorectal MDT made prior to the referral to the hepatobiliary MDT. Results The two independent hepatobiliary surgeons agreed with each other on 35/38 (92\%) of the CT scans. Our CRC MDT agreed with the hepatobiliary surgeons in $36 / 38$ (95\%) of cases. Only 6/29 (21\%) patients deemed suitable by review of the CT scan by the hepatobiliary surgeons went on to have a liver resection due to a variety of disease and patient factors.

Conclusion Our results show that our local colorectal MDT was able to make an accurate assessment of the need for referral for consideration of liver resection in this group of patients. We question the need for all similar cases to be "automatically" discussed with a hepatobiliary MDT such as those patients with wide spread liver disease. It is clear from the fact that only $21 \%$ of suitable patients for liver resection went on to have a resection that the colorectal MDT is making complex decisions based on many other patient factors.

Competing interests None declared.

\section{PWE-107 POTENTIAL ROLE OF INTRAVENOUS IMMUNOGLOBULIN TREATMENT IN THE MANAGEMENT OF PATIENTS WITH SEVERE AND RECURRENT CLOSTRIDIUM DIFFICILE INFECTION}

doi:10.1136/gutjnl-2012-302514d.107

${ }^{1,2} \mathrm{~T}$ M Monaghan, ${ }^{*}{ }^{1} \mathrm{M}$ Wilson, ${ }^{2} \mathrm{~K}$ Teahon, ${ }^{1,2}{ }^{2} \mathrm{R}$ Mahida. ${ }^{1}$ Institute of Infection, Immunity \& Inflammation, University of Nottingham, Nottingham, UK; ${ }^{2}$ Digestive
Diseases \& Thoracics Directorate, Nottingham University Hospitals NHS Trust, Nottingham, UK

Introduction Approximately $30 \%$ of patients with Clostridium difficile infection have recurrent disease, despite adequate treatment of the initial episode. Some patients with severe $C$ difficile-associated disease $(\mathrm{CDAD})$ fail to respond to standard treatment with oral vancomycin (125 $\mathrm{mg}$ three time a day). Intravenous immunoglobulin (IVIG) has been used in the management of these two groups of patients but no controlled trials have been reported and published clinical experience is mixed. We report our experience of the use of IVIG in these patients, including subsequent course of disease.

Methods Clinical information was collected prospectively and/or retrospectively (via review of case notes and hospital information system) in seven patients [median age 77 yrs (range 52-91 yrs), six female] with recurrent $\mathrm{CDAD}$ and five patients [62 (21-75) yrs, four female] with severe $C$ difficile-associated colitis. IVIG was used at a dose of $400 \mathrm{mg} / \mathrm{kg}$, rifaximin was given for 2 weeks (200 mg twice daily) and Saccharomyces boulardii for 4 weeks (125 mg four times a day).

Results Recurrent CDAD: median 5 (range 3-8) episodes of disease occurred over $9(6-15)$ months. Two patients (4 and 7 episodes) were treated with courses of vancomycin and did not subsequently have a recurrence over the following 5 and 40 months (respectively). In addition to vancomycin courses, three patients received rifaximin (two also had $S$ boulardii) and were free of recurrence over subsequent $15-27$ months. One patient (8 episodes) failed to respond to the above treatment, but after IVIG, there has been no recurrence (20 months follow-up). By contrast, a patient with myeloma did not respond to three IVIG infusions. Severe CDAD: CT showed colitis in all, CRP-107 (58-366) mg/l, WBC-17.2 (4.8-39.7) $\times$ $10^{9} / 1$. In addition to IVIG, all also had high dose oral vancomycin (500 mg four times a day) and intravenous metronidazole. Four (out of five) responded and were subsequently discharged and one had recurrence (responded to oral vancomycin). One patient, whose IVIG was delayed by $48 \mathrm{~h}$ after it was recommended, required a colectomy.

Conclusion (1) Some patients with multiple recurrences of CDAD may not require treatment in addition to courses of vancomycin but others may respond to IVIG. (2) There may be a role for IVIG in the management of patients with severe acute CDAD. (3) Our experience may facilitate identification of clinical characteristics to enable recruitment of suitable patients, with recurrent or severe CDAD, in multi-centre clinical trials of IVIG.

Competing interests None declared.

\section{PWE-108 TOXIN A-SPECIFIC ANTIGEN-ACTIVATED AND MEMORY B CELLS IN THE CIRCULATION OF PATIENTS WITH CLOSTRIDIUM DIFFICILE INFECTION}

doi:10.1136/gutjnl-2012-302514d.108

T M Monaghan, ${ }^{*}$ A Robins, H F Sewell, Y R Mahida. Institute of Infection, Immunity $\&$ Inflammation, University of Nottingham, Nottingham, UK

Introduction In Clostridium difficile infection, antibody-mediated immune response to secreted toxins $\mathrm{A}$ and $\mathrm{B}$ (which are also the main virulence factors) appears to be important in determining the nature of clinical disease. During a bacterial infection, activation of $\mathrm{B}$ cells leads to loss of immunoglobulin (Ig) D and expression of antigen-specific Ig on the cell surface. Following resolution of infection, antigen-specific memory B cells may be detectable in the circulation. Our aim was to identify circulating toxin A-activated B cells during clinical disease and toxin A-specific memory B cells following resolution of $C$ difficile infection. 
Methods Purified $C$ difficile toxin A was labelled with Alexa Fluor 488 (toxin $A^{488}$ ) and its biological activity and specificity of fluorescence were confirmed using Vero cells and anti-toxin A antibody, respectively. Peripheral blood mononuclear cells (PBMNCs) were obtained from 20 patients [13 female, 7 male; median age 67 yrs (range 32-96yrs)] with $C$ difficile infection, within 10 days of diarrhoeal onset. For flow cytometry, PBMNCs were incubated on ice in the dark for $1 \mathrm{~h}$ in the absence or presence of toxin $\mathrm{A}^{488}$. After washing, cells were labelled with anti-CD19-ECD (B cell marker) and anti-IgD-PE (to identify antigen-activated IgD-negativecells). PBMNCs were also polyclonally stimulated in vitro for 6 days to induce differentiation of memory B cells to antibody secreting cells (ASCs). Enzyme-linked immunospot (ELISPOT) assays were used to quantify toxin A-specific IgG ASCs and expressed as percentage of total IgG ASCs. Toxin A-specific IgG antibody levels in sera were studied by ELISA. Data are expressed as median (range).

Results Compared to control buffer, a significantly greater proportion of events (flow cytometry) were seen in the CD19-positive, IgD-negative gate in PBMNCs exposed to toxin $A^{488}[0.09 \%(0 \%-$ $0.54 \%)$ vs $0.92 \%(0.09 \%-1.78 \%) ; p<0.001]$. In four patients studied at the same time as flow cytometry, toxin A-specific ASCs were detected by ELISPOT assays $(0.04 \%-2 \%)$. In studies over $4(1-10)$ months after infection, toxin A-specific ASCs were observed [0.33 $(0.07-2.12) \%$. Serum anti-toxin A antibodies were detectable in eight patients at the time of clinical disease and in four patients, the antibody levels increased over the following $6(2-10)$ months.

Conclusion (1) A small population of toxin A-specific, antigen-activated $B$ cells can be detected in the circulation soon after $C$ difficile infection. (2) In addition to circulating antibody, toxin A-specific memory B cells can be detected over many months after $C$ difficile infection. (3) Future studies can investigate the relationship between the development of $B$ cell responses to $C$ difficile toxins and the nature of clinical disease.

Competing interests None declared.

\section{PWE-109 SHOULD CT COLONOSCOPY REPLACE FLEXIBLE SIGMOIDOSCOPY EXAMINATION AS FIRST LINE INVESTIGATION IN PATIENTS WITH SUSPECTED COLORECTAL CANCER?}

doi:10.1136/gutjnl-2012-302514d.109

${ }^{1} \mathrm{U} D$ Simoniuk, ${ }^{2} \mathrm{~A}$ Milewska, ${ }^{1} \mathrm{C}$ Kirre. ${ }^{1}$ Department of Gastroenterology, Southport Hospital, Southport, UK; ${ }^{2}$ Medical University of Bilaystok, Bialystok, Poland

Introduction It is recommended that all patients undergoing barium enema have a flexible sigmoidoscopy to exclude disease in rectum and sigmoid colon. With the introduction of CT colonoscopy (CT colon) is sigmoidoscopy still required for the investigation of patients with suspected colorectal cancer (CRC). ${ }^{1}$ We reviewed CT colon and flexible sigmoidoscopy results in our patients who underwent both procedures with a view to answering this question. Methods The findings of CT colon in 520 consecutive patients were reviewed by a GI radiologist blinded to the findings at flexible sidmoidoscopy. The mean age of the population was 65 years old. Patients with inadequate bowel preparation for flexible sigmoidoscopy, colonoscopy, polypectomy, abnormal MRI or CT colon as first line investigation and more than 6 months period between CT colon and flexible sigmoidoscopy were excluded. Statistical analysis was performed with $\chi^{2}$ and Fisher test.

Results 332 patients were excluded for the reasons stated above. 188 patients were analysed. In $88 \%$ of these there was concordance between the results of CT colon and flexible sigmoidoscopy. Statistically significant $(p<0.001)$ association was shown between detecting cancer by flexible sigmoidoscopy vs CT colon. The sensitivity and specificity of flexible sigmoidoscopy was $74 \%$ and $99 \%$ respectively [ppv-0.93, npv-0.94]. Flexible sigmoidoscopy did not identify six cancers which were revealed on CT colon. CT colon did not detect two cancers due to collapse colon and further investigation was recommended. There was statistical significant $(p<0.05)$ association between bowel symptoms such as PR bleeding and iron deficiency anaemia and the diagnosis of bowel cancer in patients undergoing flexible sigmoidoscopy.

Conclusion CT colon should replace flexible sigmoidoscopy especially in elderly patients as first line investigation. Flexible sigmoidoscopy still has a role in those patients who found on CT colon to have a collapse left colon.

Competing interests None declared.

\section{REFERENCE}

1. NICE clinical guideline. "The Diagnosis and Management of Colorectal Cancer". Issued: November 2011.

\section{PWE-110 WHAT IS THE SIGNIFICANCE OF "DIMINUTIVE" COLONIC POLYPS IN PATIENTS SCREENED FOR COLON CANCER AFTER A POSITIVE FAECAL HUMAN HAEMOGLOBIN TEST (FHH)?}

doi:10.1136/gutjnl-2012-302514d.110

V Mahesh, ${ }^{*}$ A Lim, K Pietris, J Argyrides. Department of Gastroenterology, Royal Adelaide Hospital, Adelaide, Australia

Introduction By definition "diminutive" colonic polyps are $\leq 5 \mathrm{~mm}$ in size. Improvements in optical imaging modalities and image resolution coupled with increased operator awareness have resulted in a higher detection rates at colonoscopy. Although such polyps are low risk for malignancy, studies suggest that high grade dysplasia may occur up to a rate of $4 \%$ in these polyps. There is an ongoing debate regarding the natural history and prognostic relevance of these polyps including suggestions for non-resection/to resect and discard. Aim To analyse the significance of "Diminutive" colonic polyps in patients screened for colon cancer after a positive Faecal Human Haemoglobin test.

Methods Patients referred to a single tertiary institution in South Australia for screening colonoscopy after detection of a positive FHH. All patients referred between 2007 and 2010 were included in this retrospective study. The patients were referred either through the National Bowel Cancer Screening Program (NBCSP) or through GP initiated screening. Polyps were measured after the tissue was placed in formalin (histological size). For each colonoscopy the predetermined aim was to resect all polyps and assess histologically. Results A total of 384 patients had colonoscopy. NBCSP referred $(\mathrm{n}=173,45 \%)$ and GP initiated FOBT ( $\mathrm{n}=211,55 \%) .228 \mathrm{M}: 156 \mathrm{~F}$ with mean age of $61.8 \pm 0.6 \mathrm{yrs}, 305$ polyps in total. Majority of the polyps $(57 \%, \mathrm{n}=173)$ were $\leq 5 \mathrm{~mm}$ compared with $6-9 \mathrm{~mm}(23 \%$,

Abstract PWE-110 Table 1 Histology of polyps $\leq 5 \mathrm{~mm}$ in size

\begin{tabular}{lrl}
\hline Tubular adenoma- low grade dysplasia & 86 & $48 \%$ \\
Tubular adenoma-high grade dysplasia & 1 & $0.6 \%$ \\
Serrated adenoma & 8 & $4.6 \%$ \\
Tubulovillous adenoma-low grade dysplasia & 10 & $5.8 \%$ \\
Tubulovillous adenoma-high grade dysplasia & 0 & $0 \%$ \\
Villous adenoma-low grade dysplasia & 1 & $0.6 \%$ \\
Villous adenoma-high grade dysplasia & 0 & $0 \%$ \\
Adenocarcinoma & 0 & $0 \%$ \\
Hyperplastic polyps & 66 & $38.1 \%$ \\
Total & 173 & \\
\hline
\end{tabular}

